\title{
COVID19 and e-learning: the Adaptive Lockdown Prototype
}

\author{
Agostino Marengo ${ }^{1^{*}}$, Alessandro Pagano ${ }^{1}$ \\ ${ }^{1}$ Economics Department (Economia e Finanza) University of Bari - Bari - Italy
}

\begin{abstract}
It was March 2020 in Italy, and the nation with all the world is facing the COVID-19 pandemic. Most countries were in lockdown, being asked to "Stay home. Stay safe". Lockdown has closed schools and universities in most countries. UNESCO estimates that over $90 \%$ of the world's students are not currently attending school in response to the pandemic, with over 1.5 billion learners affected (UNESCO, 2020). The shifting to online learning was a rapid process, and it was wideout of need. This paper will describe the development and implementation of an Adaptive System Prototype, called Adaptive Lockdown Prototype, to face the lack of e-learning innovation during the pandemic lockdown, to face the students' and teachers' necessities, managing an automated and customized learning experience. There is much literature about the pure hypothesis of personalized learning environments, but few are articles about the development of those environments. The research team's goal was to develop an adaptive learning plugin implemented on mostly used, Open Source, Learning Management Systems. The primary purpose was to maximize a students' performance during the lockdown, allowing them to manage a customized learning experience. This paper describes the main steps for the implementation of the Adaptive Lockdown Prototype.
\end{abstract}

Keywords: Adaptive learning, e-learning, Personalized Learning Environments, LMS, Open Source.

\section{Introduction}

Due to the lockdown restrictions, the lack of face-to-face contact forced teachers and students to face troubles in managing their issues in using the technology to teach and learn.

COVID19 pandemic, the lockdown, and the new way to live our social life have changed the mindset and pushed everybody to think differently about learning models and the technology to support them.

Therefore, it is crucial to extend the effective use of technology-enhanced learning radically, to face the lockdown problems and other pandemic issues that will limit the education process inside universities and schools. It aims to promote and support a new learning paradigm focused on the experience building using collaborative learning approaches in a ubiquitous, collaborative, experiential-based, contextualised, individualised, and personalised way and taking into account informal learning aspects. 


\section{3rd International Conference on Advanced Research in Teaching and Education}

Researchers believe that adaptive learning is an essential requirement to improve the teaching quality and students' performance throughout the learning strategy. An adaptive learning element enhances the starting skills of a student, tailoring an appropriate learning path with specific content related to the student's learning style. This solution will improve the students' performance during the lockdown period, enhancing the engagement required to cultivate the interest and passion in studying the proposed content for each student.

The route to follow in order to build a significant learning and teaching performance during the lockdown, cannot ignore the element of personalized learning environments.

The goal to generate a customized learning path for any learner provides a challenging work for researchers involved, both, in informatics and education specializations.

Furthermore, to develop a customized (adaptive) learning path means that student's starting skills should be assessed for delivering a tailored learning path appropriate for each student.

Different learners may have distinct characteristics, previous experience, different background, starting talents, motivation or necessities. The research group thinks that it is essential to design and produce adaptive educational systems which consider the several uniqueness of each student, in order to support chances of professional and educational growth.

This research study aimed to identify an approach to produce and supply the right content to the student's according to their learning style and to implement it on an Open Source LMS designing a tool called Adaptive Lockdown Prototype.

The goal of this research is to develop an adaptive technology, based on an Open Source LMS that automatically build up a suitable learning path for each student considering his assessed profile [10].

The research project aims to launch an innovative Intelligent Agent to support e-learning strategies and develop such an approach, evaluating the impact on the speed and success of learning given specific learning outcomes.

The plug-in prototype will be delivered on the Open Source Community using GNU License to let them spread this innovation and enhance the e-learning possibilities during lockdown periods.

\section{The Methodology}

How could adaptive learning be executed in Open Source LMS to build up a learning path appropriate for each user selecting the right learning objects? On the methodological point of view, this one is the central problem that this research project aspires to solve designing a Tool with an adaptive technology structure.

Therefore, to answer this problem, the research team explored the adaptive technology and defined the implementation of an adaptive "strategy" in the most used Open Source Learning Management System (LMS): Moodle.

As we all know, each student may have different traits, previous experience, starting aptitudes, goals or needs. This variety commonly needs the production of different information to different learners in different formats. 


\section{3rd International Conference on Advanced Research in Teaching and Education}

Information should be adjusted to what a student already knows (prior knowledge) or can do (prior skill), and it depends on his learning capabilities. Moreover, information should adapt to students' learning preferences or style, and it depends on his performance level, interests, personal circumstances and motivation.

In every innovation project, it has been crucial to perform an in-depth study of the state of the art; in fact, this was the first step in building up an adaptive learning tool.

Then, the second step has been to recognise the right profile of the students, with their learning style and starting skills in order to determine the appropriate learning content that corresponds to the individual students learning style and learning needs.

Once the student's "learning profile" was defined, the third step in the presented methodology approach was the development of an Adaptive Learning Path using the learner's behaviour patterns, like learning styles, goals and performance.

At the end of this phase, the research team proceeded to the code development of the software and all the technical works needed to meet the functional requirements of the Adaptive Lockdown Prototype.

The expected result was to give the students a useful tool to reach higher motivation and finish the defined learning path during a lockdown period, avoiding the situation in which they have to study unsuitable contents, losing the right motivation to reach their goals in their education process.

After the development phase, a stage of tests and experiments conducted in vitro and in vivo will be launched. This step will ensure compliance with the objectives of the project and measure the degree of goals achievement, through statistical surveys and satisfaction questionnaires on teachers and the learners.

\section{Adaptive LMSs For Pandemic}

Today, the Learning Management System turns into a complete and elaborate application framework; several methods to different adaptive systems have been designed in the last few years with differing success grades [7] [8] [9]. Nevertheless, not much work has been done to generate active and dynamic learning environments that adapt to the student's specific learning needs and can actively support and enhance learning scores towards specified results during the pandemic restrictions.

The overall intent of an LMS is to feed students with information as well as with practical opportunities in order to help them to acquire specific competencies and to increase their operational knowledge about a studied topic or to achieve specific learning goals.

Moodle, a well-known open-source Learning Management System (LMS), can be employed to provide online learning in a combination of environments like virtual schools, K12 [4] [5], higher education, corporate universities, to name but a few. Moodle is designed to be utilised mainly as an asynchronous learning tool with the following interactive features.

Forums, for example, could be structured in distinct ways and can include peer rating of each posting. Furthermore, a lesson delivers stimulating and adjustable content and wiki, in general, enables documents to be authored collectively in a simple markup language using a web browser. 


\section{3rd International Conference on Advanced Research in Teaching and Education}

Grades/tests aside, Moodle lacks student classification, so the research group focused its work designing a student modelling approach to add a new feature: adapted learning goals.

Adaptive learning systems have traditionally been divided into separate components or 'models'. While different model groups have been presented, most systems include some or all of the following [3] [8] [9]:

- $\quad$ Expert model - with the knowledge which is to be trained.

- $\quad$ Student model - which tracks and learns around the student style.

- Instructional model - which conveys the knowledge.

- Instructional environment - the user interfaces for interacting with the system.

\subsection{The Sutent Model}

The traditional e-Learning solutions are mainly based on the information transfer approach and, in most cases, they are distance learning platforms able only to manage content and present them to the students that, passively, consume it. In other words, the current e-Learning solutions take care only of the inputs of the whole learning process (e.g. the educational resources), and they suffer from relevant absences like the support for pedagogies, the contextualization of learning, the learner centrality and activity, lowers the level of effectiveness of the human learning [2].

More complex solutions, instead, are focalized on a specific educational model, and this means to superimpose to learners how they have to learn without taking into account their dispositions or preferences and to develop pedagogically closed solutions, that constrain learners to learn and teachers to teach following a prearranged method.

The student model includes all relevant information that the system has gathered about the learner and can build and update during the process called "student modelling". There are two different ways of student modelling: collaborative and automatic student modelling.

In the collaborative approach, an often-used technique is to let students fill out a test in order to get information about their learning styles or let the students provide explicit feedback which can be used to build or update their model or each one of them could be allowed to update the information of the student model directly.

However, the automatic approach should consider the process of building up the student model automatically, reasoning on the behaviour of the learners during their use of the system, while the main problem is to build a robust student model with reliable information. A solution might be the use of different, more trustworthy references, such as the results of tests during the learning process [2].

Furthermore, we need to consider a static or dynamic student modelling. Static will be done when the student model is initialised only once. Dynamic approach will be done when the system frequently updates the information in the student model.

\subsection{Adaptivity}

Adaptivity can be provided once information about the learners is available from the student model. 


\section{3rd International Conference on Advanced Research in Teaching and Education}

Different methods determine which features of the system are different for different learners, in order to provide students with adapted courses, and those features can be classified into two main groups, namely for adaptive presentation and adaptive navigation support.

The first one, "Adaptive presentation", includes features based on content such as adaptive multimedia production, adaptive text exposition, and also adaptation of the modality.

However, the second one, called "Adaptive navigation" includes some different components such as direct suggestion, map adaption, as well as adaptive generation of links [1].

An example of adaptive navigation support in Moodle is the Activity locking in which the system will lock a student out of a specific activity until the he satisfies a score, a view criterion matrix for one or more specific activities/resources in a course.

Some specialised conditional activity locks are based on an overall course criterion matrix [7]. With this tool, the teacher is the only one who can change or hide/unhide activities and resources depending on his/her assessment of the individual differences of his/her students. So, teachers or instructors, who are creating a course, should prepare some alternative version of the course for better or weaker students.

A much more effective approach it's an adaptive sequencing of learning courses/activities for each particular learner based on a student model updated after each interaction with the learner.

The student takes some sort of quiz/survey, etc. each time he registers to a learning goal. Based on test's results, only the activities the student needs to complete will appear in the course.

\section{The Adaptive Prototype}

In order to design a customized learning path and learning experience, it is essential to use data mining methods and tools to help the teacher analyze the results.

Moodle Data Mining Tool [3] [4] [5] needs experience in data mining for practical use. It needs to develop an automated process that could help teachers take the right information without technical operation, neither choosing algorithms nor parameters and with good customization of the resulting layout.

The plugin developed, dynamically changes what the student catches as links of activities and resources, working on the dynamic combination and sequencing of the set of learning objects in the course environment.

The combination process indicates:

- $\quad$ The learning objects are already designed and produced as reusable; they are already in the course and only require to be hidden/unhidden.

- The learning objects determined as needed by the student, based on some assessments, can be picked up from the repository and installed.

- $\quad$ Any other learning object identified as dependency or prerequisite may also be installed automatically and shown to that individual student as the dependency/prerequisite information is stored as metadata.

The combination process implies that conditions and locks in the learning path will change automatically by some assessment checks. 


\section{3rd International Conference on Advanced Research in Teaching and Education}

As a matter of fact, the team chose to add the option to link a test to a specific learning object and/or learning goal. The test every student takes when enrols to a learning goal is the sum of tests linked to learning objects, which are prerequisites of the goal's learning objects. If the student passes a specific test, the learning object linked to it will be hidden to him [14].

At the end of every learning goal, the student must pass a test, after which every goal's learning object will be hidden to him. When the student enrols to a new learning goal, he does not have to make a test linked to learning objects hidden to him [12] [13] [15].

To accomplish this task in the project, the team has added some tables to the Moodle database, using an old research project [1] and enhancing it with some new tables and features.

New Features in Moodle management tool:

1. Manage learning goal: a fresh new page to let the teacher manage a learning goal and link a test to it. Activities in this page update learning_goals, mng_contents and lg_tests tables.

2. Link test: an update to "create/modify learning objects" in Moodle with the option to link the learning object to a premade test. This updates the lo_tests table.

3. Count prerequisite: same page as point 2, with the option to choose the right learning objects as a prerequisite. This updates the lo_prerequisites table.

4. Final tests update: at the end of each test, if the student has been successful, the hidden_los table is updated.

New Tables in the Moodle database:

- learning_goals: all learning goals are recorded in here.

- $\quad$ mng_contents: about the content, every learning goal is made.

- $\quad$ mng_enrolments: shows which user is enrolled to each learning goal.

- $\quad$ mng_tests: learning goal links to each test.

- $\quad$ hidden_los: stands for hidden learning objects.

- lo_prerequisites: learning object prerequisites.

- lo_tests: learning object links to test.

\section{Conclusions, validation and future developments}

In this paper, we have presented our approach to the analysis and design of an Adaptive Learning System for formal learning to face the lockdown problems in inclusion, engagement and performance. The research work has been mainly motivated by the difficulties we have encountered in exploiting current methodologies and tools in order to model the problem domain.

The required personalization individualized monitoring of the learners, which plays a vital role because without information on the progress and the results obtained by the learners, it will be challenging to support and control their behaviours. This monitoring requires "intelligent" components to detect in real-time the learner's profile and learning preferences in order to adapt their learning automatically at each step of the learning process. 


\section{3rd International Conference on Advanced Research in Teaching and Education}

The team believes that the proposed approach can also be exploited in other domain, different from the e-learning context, where requirements do not directly come from the end-user but mainly from a set of models and processes derived from theoretical researches.

Future works on the architecture consist of refinement of the prototype and to conduct several "in vitro" and "in vivo" testing phase in a pandemic lockdown simulated environment, to achieve a significant number of data and define the final Adaptive Lockdown Methodology based on the massive use of the developed Adaptive Prototype.

Some new perspectives will be open shortly to improve the validation process of the Adaptive Lockdown Prototype, evaluating the different variables showed up during the lockdown and post-lockdown period.

The "in vitro" and "in vivo" testing phases are crucial to investigate about significant enhancements of the students' performance and, above all, significant reduction of the "at-risk" students' percentage.

This significant reduction will exist due to some different variables that could have different weights in the final results; one of the more significant variables is the lockdown period, in which the students have changed their method to approach the study.

\section{References (TNR 14pt., bold)}

(Book style - Author, year. Title (in italics). Publisher, location of publisher.)

[1] Marengo, A., Pagano, A., Barbone, A. 2012 - Adaptive learning: A new approach in student modeling - Proceedings of the International Conference on Information Technology Interfaces, ITI

[2] Jack P. Krichen, Investigating learning styles in the online educational environment Proceedings of the 8th ACM SIGITE conference on Information technology education, October 18-20, 2007, Destin, Florida, USA

[3] Marengo, A., Pagano, A., Barbone, A. 2013 - Data mining methods to assess student behavior in adaptive e-learning processes - Proceedings - 2013 4th International Conference on e-Learning Best Practices in Management, Design and Development of eCourses: Standards of Excellence and Creativity, ECONF 2013

[4] Marengo, A., Pagano, A., 2020 - Innovative Ways to Assess Soft-skills: The in-Basket Game Online Experience - Proceedings of the European Conference on e-Learning, ECEL - 2020, October

[5] Marengo, A., Pagano, A., Ladisa, L. 2016 - Mobile gaming experience and co-design for kids: Learn German with Mr. Hut - Proceedings of the European Conference on e-Learning, ECEL - 2016-January, pp. 467-475

[6] Marengo, A., Pagano, A., Ladisa, L. 2018 - Towards a mobile augmented reality prototype for corporate training: A new perspective - Proceedings of the 14th International Conference on Mobile Learning 2018, ML 2018 - pp. 129-135

[7] Minguillón, J., Conesa, J., Rodríguez, M. E., \& Santanach, F. (2018). Learning analytics in practice: providing E-learning researchers and practitioners with activity data. In Frontiers of Cyberlearning (pp. 145-167). Springer, Singapore. 


\section{3rd International Conference on Advanced Research in Teaching and Education}

[8] Rakic, Slavko, Pavlovic, Marko, Softic, Selver, Lalic, Bojan, Marjanovic, Ugljesa. (2019). An Evaluation of Student Performance at e-Learning Platform. In 17th International Conference on Emerging eLearning Technologies and Applications (ICETA) Emerging eLearning Technologies and Applications (ICETA), 2019 17th International Conference on. :681-686.

[9] Raluca-Giorgiana CHIVU, Gheorghe ORZAN, Ionuț-Claudiu POPA, 2019. Education Software and Modern Learning Environment: Elearning. In Proceedings The 15 th International Scientific Conference eLearning and Software for Education.

[10] BUTUM, Lavinia Cornelia; STAN, Sergiu Octavian; GĂITĂNARU, Andrei, (2019). Tools Used in eLearning and Specific Risks Associated with This. Enabling Students, Professors and Researchers with ICT Skills. In eLearning \& Software for Education.

[11] PANFIL, Georgică. (2018). A Comprehensive Analysis of the Students' Perception Referring to the Elearning Platform. In eLearning \& Software for Education.

[12] Rakic, Slavko, Tasic, Nemanja, Marjanovic, Ugljesa, Softic, Selver, Lüftenegger, Egon, Turcin, Ioan. (2020). Student Performance on an E-Learning Platform: Mixed Method Approach. In International Journal of Emerging Technologies in Learning; 2020, Vol. 15 Issue 2, p187-203, 17p.

[13] Rakic, S., Softic, S., Vilkas, M., Lalic, B., Marjanovic, U. (2018). Key Indicators for Student Performance at the E-Learning Platform: An SNA Approach. 16th International Conference on Emerging eLearning Technologies and Applications (ICETA) Emerging eLearning Technologies and Applications (ICETA), 2018 16th International Conference on.

[14] Marienko, Maiia, Nosenko, Yulia, Sukhikh, Alisa, Tataurov, Viktor, Shyshkina, Mariya. (2020). Personalization of learning through adaptive technologies in the context of sustainable development of teachers education. In Computer Science, Physics.

[15] Martin, Florence, Chen, Yan, Moore, Robert L., Westine, Carl D. (2020). Systematic review of adaptive learning research designs, context, strategies, and technologies from 2009 to 2018. Educational Technology Research \& Development. Aug2020, Vol. 68 Issue 4, p1903-1929. 27p. 